\title{
Enhancement of Online Course Development based on a Public Cloud Computing Infrastructure
}

\author{
B.Veeramallu \\ Associate professor \\ CSE dept \\ Kl university
}

\author{
Konda Navya \\ First year M.Tech \\ CSE dept \\ KI university
}

\author{
Bhamidipati Pavani \\ First year M.Tech \\ CSE dept \\ Kl university
}

\begin{abstract}
There are several websites which offer a user with several courses. But the current Online Course structure had failed because of several reasons. Firstly the user have to pay, if the server is down user cannot access the website. The major problem was infrastructure and 24/7 support. To overcome the short comings deploy the website and use throughout the world via internet. This initiates requirement of a cloud. This paper will help in overcoming the drawbacks of the existing system by developing a Online Course model by adding extra features like uploading documents by the professors, viewing video lectures, downloading documents, asking questions, giving answers to the questions, discussing contents of courses among students, etc. and deploying that on Microsoft's cloud using MS Azure.
\end{abstract}

Keywords: Cloud, Public, Windows Azure, Resources, Services, Online, learning, Course.

\section{INTRODUCTION}

Computers play a prominent role in the educational sector, as well as in business and industrial sectors. In educational field a professor can keep information on cloud to share among the world. A professor can find a new concepts in a course; he/she will get the idea of the new concepts, how to solve the problems and all the information about the concept. The professor can place the information on the cloud. If some problem came to student about the concept then that professor can get information about the problem and professor can solve the problem for the student. With the help of cloud computing the students in different countries can get information about different courses. The information in cloud will be encrypted [1] for the security purpose, so that only the authorized one's can use the data in the cloud. Windows Azure is Microsoft cloud computing platform used to build the applications deploys the applications and manage the applications through a global network of Microsoft managed datacenters. Windows Azure allows applications to be built using many different languages, with many different frameworks and tools. Windows Azure provides both Platform as a Service and Infrastructure as a Service services and is classified as the "Public Cloud" in Microsoft's cloud computing strategy, along with Microsoft Online Services. SQL Azure is cloud based service from Microsoft data storage capabilities as a part of the Azure Services Platform.SQL Azure allows users to make relational queries against the stored data, which can be either the structured or the semi structured, or even the unstructured documents. SQL Azure performs querying data, search, and data analysis and data Synchronization [6].

\section{CLOUD COMPUTING}

A cloud is a virtual space which is available for the users to deploy their applications. A cloud services are having three distinct characteristics that differentiate it from traditional hosting they are, it is sold on demand, typically by the minute or the hour; it is elastic so that the user can have as much or as little of a service as they want at any time; and the service is fully managed by the cloud provider so that customer need not to do anything, if he has a computer and internet access it is sufficient. Significant innovations in virtualization and in the distributed computing, as well as it improved access to high speed Internet and a weak economy, have accelerated interest in the cloud computing. Cloud technology [2] wills provides computation, software, data access, and the storage services that do not require any end-user knowledge of the physical location and configuration of the system that delivers the services. Cloud technology providers deliver applications through internet, which are accessed from web browser, while business software and data are stored on servers at a remote location. Cloud computing [4] is the highly scalable and creates virtualized resources which can be made available to the users. Users do not require any special knowledge about the concept of Cloud computing to connect their computers to the server where applications have been installed and use them. Users can communicate among themselves through Internet with remote servers. These remote servers can then exchange their computing slots among themselves. Cloud computing is one of the new technologies [3] likely to have a significant impact on the teaching and also in learning environment.

\section{TYPES OF CLOUD SERVICES}

In cloud technology the information is shared from clients to the organization through the virtual data centers. This virtual data centers has all the required information. The cloud technology model includes three services [1], they are:
1) SaaS (Software as a service)
2) PaaS (Platform as a service)
3) IaaS (Infrastructure as a service) 


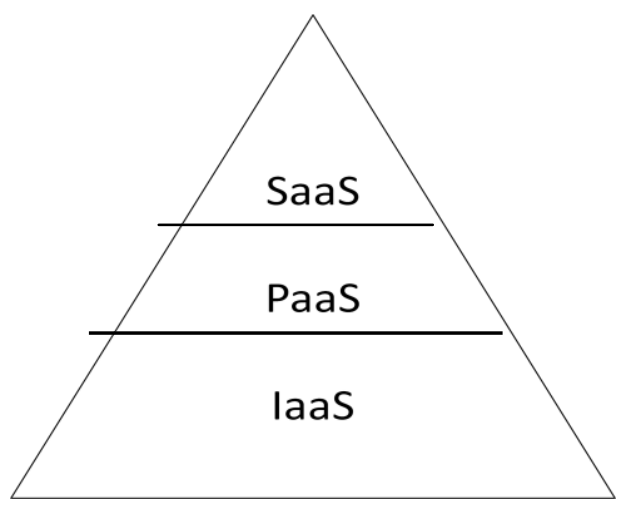

Fig 1:- Services of Cloud

\subsection{SaaS (Software as a Service):}

Saas is the cloud vender who is providing the given piece of software we want to use on their servers. It is a way of delivering applications over the Internet as a service. Instead of installing the software and maintaining software, we can simply access it through Internet, freeing ourselves from complex software and also from hardware management. SaaS [2] applications are also called Web-based software, or demand software, or hosted software. Whatever the name may be, SaaS applications run on a SaaS [5] provider's servers.

\subsection{PaaS (Platform as a Service)}

To follows the heels of SaaS, platform as a service (PaaS) [2] is another application delivery model. PaaS provides all the resources which are required to build applications and services completely from the internet without having to download any software or to install any software. PaaS services include application design development, testing, deployment, and hosting. It provides infrastructure on which software developers can build new applications or extend existing applications without the cost and complexity of buying and managing the underlying hardware and software and provisioning hosting capabilities. PaaS generally offers some support to help the creation of user interfaces, and it is based on HTML or JavaScript.

\subsection{IaaS (Interface as a Service):}

IaaS offers remote delivery of an entire computer infrastructure. Managed hosting and development environments are the services included in IaaS [2]. The user can buy the infrastructure according to the requirements at any particular point of time instead of buying the infrastructure that might not be used from months. IaaS operates on a "Pay as you go" model ensures that users pays for only what they are using.

\section{TYPES OF CLOUD COMPUTING}

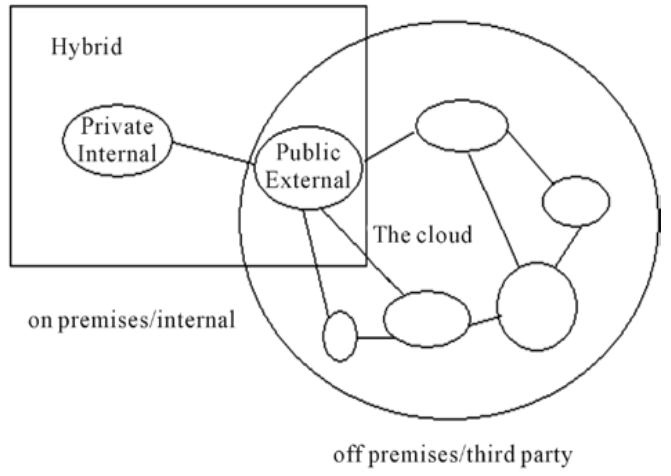

Cloud Computing Types

Fig 2: Types of Cloud Computing

\subsection{Public Cloud}

Public cloud describes cloud computing the in traditional mainstream sense, where by resources are dynamically provisioned on a fine grained, self-service basis over the internet through web applications or web services, from an offsite third party provider who shares resources and bills on a fine grained utility computing basis. A public cloud is the one which is based on the standard cloud computing model, in which the service provider makes resources, such as applications and storage, available to general public over Internet. Public cloud services may be free or may be offered on a pay-per-usage model.

\subsection{Private Cloud}

Private cloud is used within the organization or corporation. Public cloud provides hosted services to very limited number of people behind the firewall. Advances in the virtualization and distributed computing have allowed corporate network and datacenter administrators to effectively become service providers that meet the needs for their "customers" within the corporation.

\subsection{Hybrid Cloud}

Hybrid cloud is a cloud computing environment in which an organization provides and manages some resources in-house and has others provided externally. For example, if an organization might use a public cloud service, such as Amazon's Elastic Cloud for general computing but store customer data within its own data center. Although cloud computing is often said to be the future of industry, the hybrid cloud model is more prevalent for a number of reasons. Large enterprises of ten already have substantial investments in the infrastructure required to provide resources in-house. Furthermore, many organizations would prefer to keep sensitive data under their own control to ensure security [1]. 


\section{METHODOLOGY}

The proposed system is designed in such a way that the user through a browser visits courses offered and interacts with data and applications store and run in the cloud to complete a certain amount of learning activities. Here users are professors and students. Students take a particular course, get trained, interact with the professors, professors discuss with the students using discussion forum. Provide online discussion areas for Question \& Answers, exchanging and sharing learning [9] resources among students and between students and professors. The admin registers the professor, uploads the video lectures, post latest notifications, announcement and other dynamic information. The registered professors can upload the materials related to their course and have online discussion with their respective students. Students can select only a single professor, rate the professor according to the material provided by him, read the documents provided by the professor, download those documents, view the video lectures posted. Students can ask questions, giving answers to the questions, discussing contents of courses among students, finally can do online discussion with the selected professor. Online Course Development Based on a Public Cloud Computing Infrastructure model needs deployment and constant monitoring and periodic updates of database. The admin will be facilitated to manage the database on one hand and the student community, through the cloud will be accessing the data on the other hand. Admin monitors the whole project, updates the data timely.

\section{IMPLEMENTATION}

\subsection{Existing System}

The existing system makes the world of learning, completely online. There are several websites which offer a user with several courses. But the current Online Course structure [7] had failed because of several reasons. Firstly the user have to pay, if the server is down user cannot access the website. The major problem was infrastructure and 24/7 support.

\subsection{Proposed System}

To overcome the short comings deploy the website and use throughout the world via internet. This initiates requirement of a cloud. This will help in overcoming the drawbacks of the existing system by developing a Online Course model by adding extra features like discussion board, student can select the course, student can select the professor, asking questions, giving answers to the questions by professor, uploading documents by the professors, selecting the professor, downloading documents by students, viewing video lectures, rating the professor, student can communicate with another student, discussing contents of courses among students, finally can do online discussion with the selected professors,etc. and deploying that on Microsoft's cloud using MS Azure.

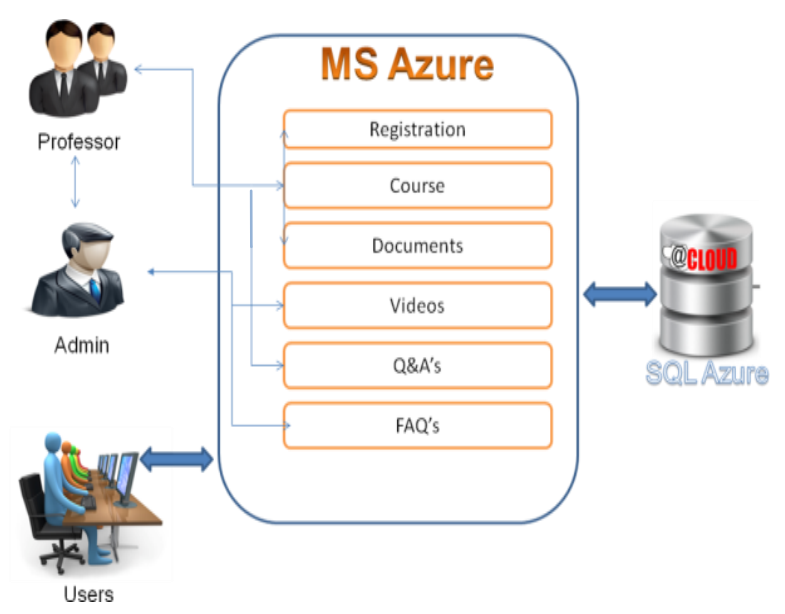

Fig 3: Deploying Online Course Development Based on a Public Cloud computing infrastructure.

\section{CONCLUSION}

In this paper we introduced a cloud computing technology in Online Course. Education is transformed with the help of Cloud computing throughout the nation. An entire world of knowledge can now be made available to teachers and students through cloud based services that can be accessed anytime, anywhere, from any device. By helping countries worldwide, lowering the cost and simplifying the delivery of educational [8] services, cloud computing [10] enables students across the globe to acquire the 21st-century skills and training they need to compete and succeed in the global information society. Online Course Development Based on a Public Cloud Computing Infrastructure is the resources and procedures storaging and running on cloud share the same human-computer interaction interface in this case, is the complexity of its internal structure is transparent to the terminal students. This approach is clearly more flexible framework to achieve easier and cheaper. It is not possible to develop a system that makes all the requirements of the user. User requirements keep on changing as the system is being used. Future enhancements that can be done to this system are:

1) Provides an opportunity that student can select more than one professor.

2) Provides online discussion among professors.

\section{REFERENCES}

[1] V. Sreenivas and C. Narasimham, "Enhancing the Security for Information with Virtual Data Centers in Cloud" Future Wireless Networks and Information Systems, Vol. 143, 2012, pp. 277-282.

[2] V. Sreenivas and C. Narasimham "A Cloud Model to Implement SaaS," Advanced Materials Research, Vol. 341-342. 2011, pp. 499-503

[3] Tejaswi Avula , Radhika Gudapati ${ }^{1}$, Manoj Kumar Nela1, Sreenivas Velagapudi "Efficient Use of Cloud Computing in Medical Science", American Journal of Computational Mathematics, Vol.2.2012, pp. 240-243

[4] B. Hayes, "Cloud Computing," Communications of the ACM, Vol. 51, No. 7, 2008, pp. 9-11. 
[5] Frank Gens, IT Cloud Services User Survey, pt.3: What Users Want from Cloud Services Providers, October 2008

[6] ^abcdf "Microsoft SQL Services FAQ". Microsoft. Retrieved 2009-04-16.

[7] Ruan Gaofeng and Cai Jing. Online Course Development Based On a Public Cloud Computing Infrastructure, Networking and Digital Society (ICNDS), Vol. 2, pp. 4750, 2010.

[8] Bo Wang and HongYu Xing, the Application of Cloud Computing in Education Informatization, Computer
Science and Service System (CSSS), pp. 2673-2676, 2011.

[9] Al-Zoube, M., El-Seoud, S.A., Wyne, and M.F.: Cloud computing based e-learning system. Intl. Arab Journal of e-Technology 8(2), 58-71 (2010)

[10] Sulistio, A., Reich, C., Doelitzscher, F.: Cloud Infrastructure \& Applications - CloudIA. In: Jaatun, M.G., Zhao, G., Rong, C. (eds.) Cloud Computing. LNCS, vol. 5931, pp. 583-588. Springer, Heidelberg (2009) 